\title{
Investigation of dynamic acting of a technological equipment on a building construction
}

\author{
Pavel Polach ${ }^{1, *}$ and Michal Hajžman ${ }^{1}$ \\ ${ }^{1}$ Research and Testing Institute Pilsen, Tylova 1581/46, 30100 Pilsen, Czech Republic
}

\begin{abstract}
This paper deals with the analysis of acting a dynamically loaded technological equipment on a building construction. The Cyclone 18 cyclotron was selected as a demonstrator of the technological equipment. Two cyclotron loading modes are simulated: excitation by a harmonic sinusoidal force and a seismic excitation. Mounting the cyclotron in the building construction is considered absolutely rigid. To create the cyclotron finite element model and to calculate the cyclotron dynamic acting on the building construction the ANSYS software was used, in which the cyclotron calculation model including its concrete chamber was created.
\end{abstract}

\section{Introduction}

Dynamic response of structures can be affected by various influences related to their operational conditions. One of very important phenomena with respect to structural safety is seismic excitation and consequent seismic response of a structure. It is an issue mainly in nuclear engineering where structural safety during a seismic event is a crucial requirement put on designers. Many publication deals with the seismic response of nuclear reactors (see e.g. [1]), while an employed modelling methodology is mostly based on the finite element method (see [2] for the application on the full-scale boiling reactor). More general structural application considering walls and slabs can be found in [3].

This paper deals with the analysis of acting a dynamically loaded technological equipment on the building construction. The Cyclone 18 cyclotron was selected as a demonstrator of the technological equipment. There exist other works dealing with specific analyses performed with cyclotron devices. Heating of the ITER Electron Cyclotron is studied in [4]. Its seismic analysis is investigated in [5] and its mechanical properties are discussed in [6].

Two cyclotron loading modes are simulated: excitation by a harmonic sinusoidal force and a seismic excitation. Mounting the cyclotron in the building construction is considered absolutely rigid. To create the finite element model (FEM) cyclotron model and to calculate the cyclotron dynamic acting on the building construction the ANSYS software was used, in which the cyclotron calculation model including its concrete chamber was created (see

\footnotetext{
* Corresponding author: polach@ vzuplzen.cz
} 
Fig. 1 and Fig. 2). The model is based on discretization of bodies using the finite element method. Mainly construction drawings served as a source material for the creation of the cyclotron FEM model.
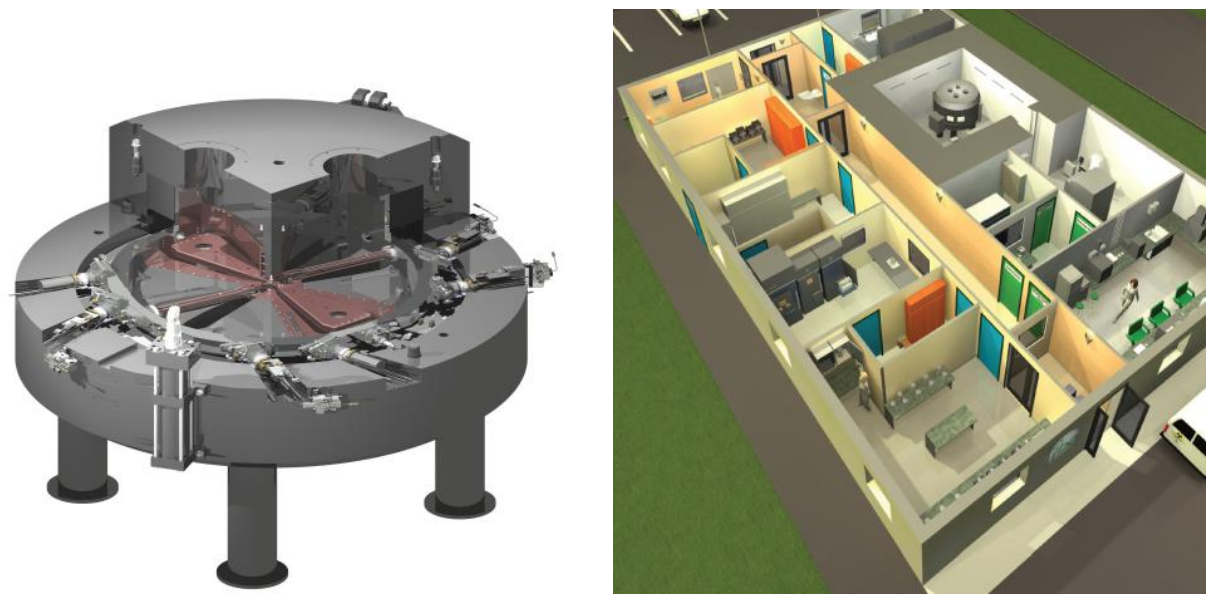

Fig. 1. The Cyclone 18 cyclotron (left) and a scheme of its location in the building (right).

\section{Calculation model of the cyclotron and its chamber}

Calculation FEM model of the cyclotron and its chamber is of a fully parameterized geometry, which enables to perform operational calculations with the modified parameters. A finite element grid of the cyclotron FEM model can be seen in Fig. 2 left in which there are simultaneously shown the places where the finite element grid of the cyclotron model was connected with the finite element grid of the chamber model applying fixed connection. The finite element grid in the section of the whole model is in Fig. 3.
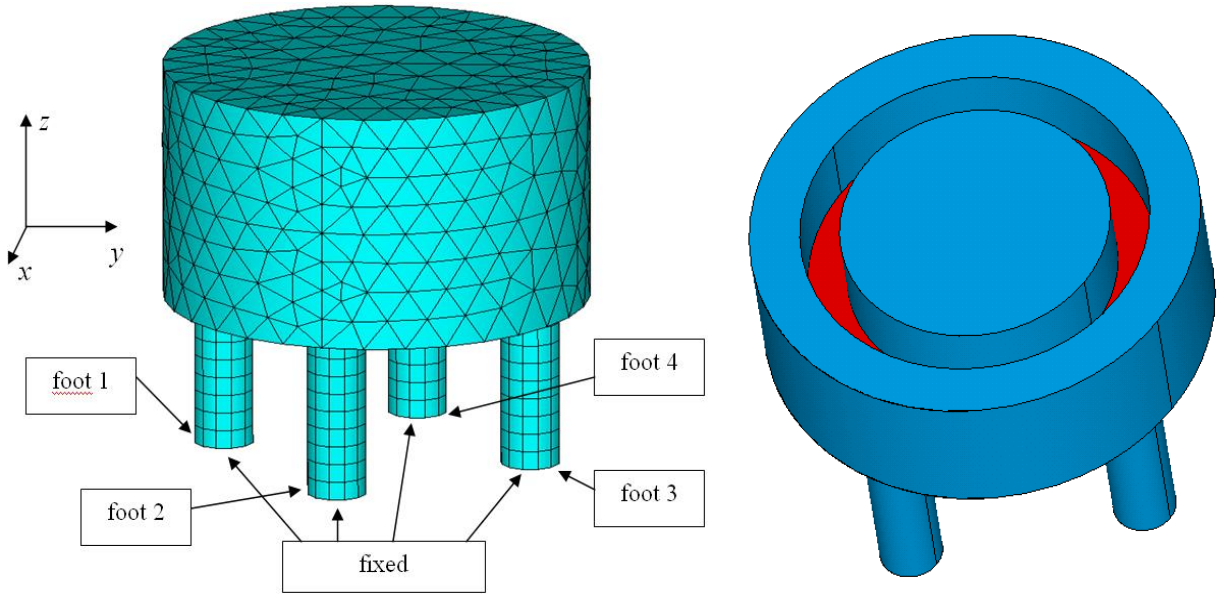

Fig. 2. The finite element grid of the cyclotron and depicting of the places with boundary conditions (left) and cyclotron section (right; harmonic excitation force acts on a red area).

Material properties used for the cyclotron model correspond to a common steel (the Young modulus $E=2.1 \cdot 10^{11} \mathrm{~Pa}$, the Poisson ratio $v=0.3$ ). The steel density value was modified that the total mass of the cyclotron in the FEM model should be identical with that 
in the construction documentation, which is a prerequisite for the calculation of the model real responses to a dynamic excitation.

Internal structure of the cyclotron was simplified for two reasons:

1. Available documentation did not enable to model internal parts of the cyclotron precisely.

2. The modelling of the cyclotron internal structure in detail would influence the results of dynamic calculations only minimally.

According to the source data from the construction documentation, the used concrete density was $2600 \mathrm{~kg} \cdot \mathrm{m}^{-3}$ for the upper and the lateral parts of the chamber and $2400 \mathrm{~kg} \cdot \mathrm{m}^{-3}$ for the bottom part of the chamber. The Young modulus $E=3 \cdot 10^{10} \mathrm{~Pa}$ and the Poisson ratio $v=0.2$ were chosen on the basis of generally known pieces of knowledge on a concrete.
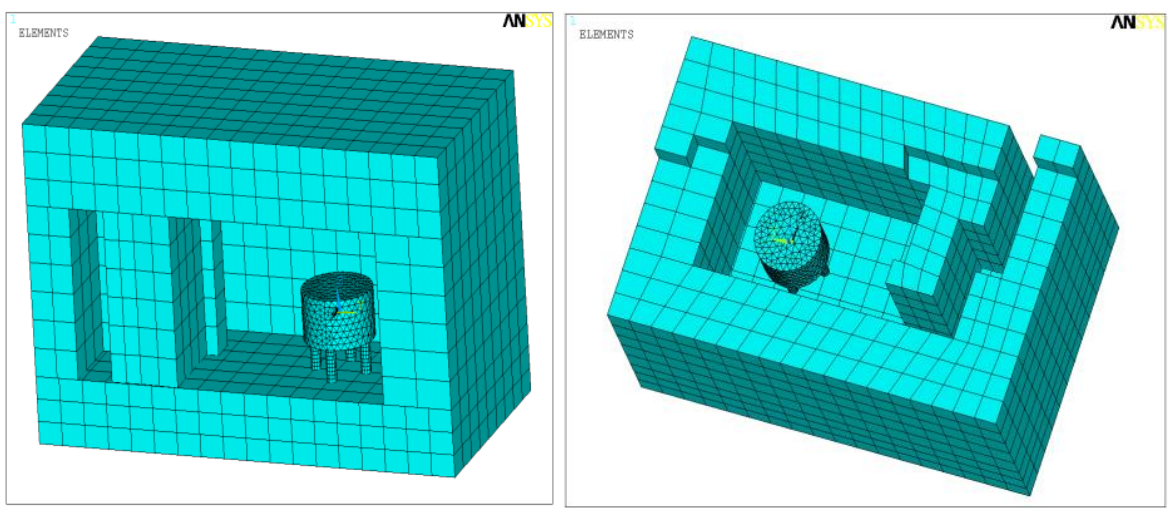

Fig. 3. Finite element grid section of the concrete chamber and the cyclotron: front view (left), view from above (right).

\section{Dynamic excitation of calculation model of the cyclotron and its chamber}

Response of a mechanical system to two types of a dynamic excitation was solved. In the first case the dynamic excitation of the cyclotron calculation model was chosen as the excitation by a harmonic sinusoidal force. In the latter case the chamber was loaded with a seismic excitation the acceleration spectrum of which issued from the proven acceleration spectrums in seismically middle active zones.

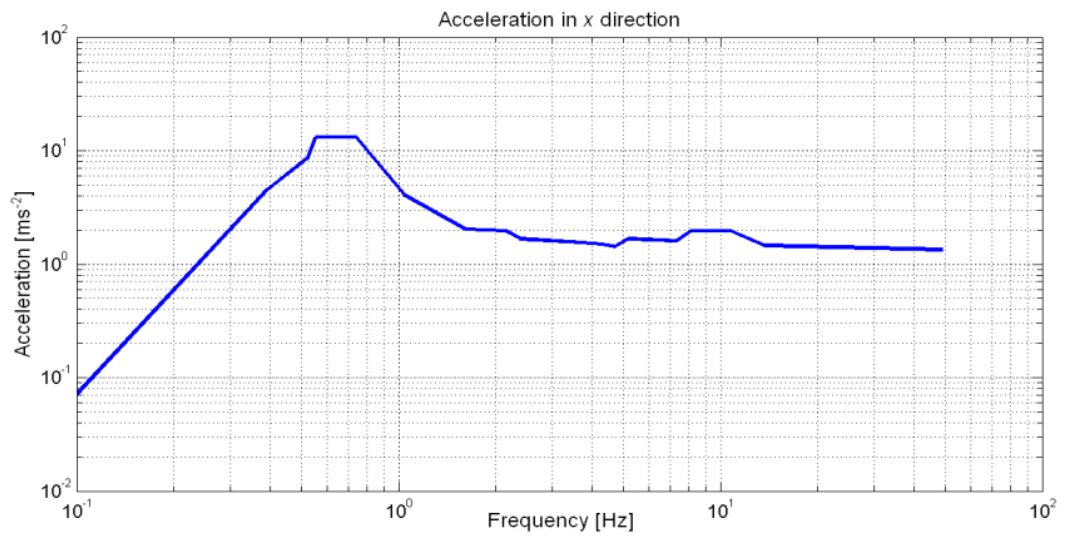

Fig. 4. Definition of the chamber seismic excitation in $x$ axis. 
Excitation frequencies in the range of $10 \mathrm{~Hz}$ to $400 \mathrm{~Hz}$ were considered at loading with a harmonic excitation force. Amplitudes of the excitation force were chosen in the range of $1000 \mathrm{~N}$ to $20000 \mathrm{~N}$. The excitation force acted in a vertical direction ( $z$ axis - see Fig. 2). Regarding the presupposition (linear behaviour of material, damping, etc.) responses should be proportionate to the chosen amplitude. This fact is confirmed by the results given in Table 1. Fig. 2 right presents the cyclotron section. The area of acting the harmonic excitation force is highlighted in a red colour, there.

Seismic loading was set on the chamber location (on its bottom part) using acceleration spectra in directions of all axes of the coordinate system ( $x, y$ and $z$ - see Fig. 2). The acceleration spectrum acting in $x$ axis direction is shown in Fig. 4.

\section{Results of dynamic calculations}

Results of the calculation of the Cyclone 18 cyclotron dynamic excitation by the harmonic sinusoidal force are given in Fig. 5 and in Table 1. The problem was solved in the whole FEM model of the cyclotron and its chamber as harmonic analysis (analysis of a steadystate vibration at monofrequency force excitation). In this chapter there are given the maximum values of dynamic forces. When calculating the maximum values of the total forces it is necessary to add a reaction force of $60 \mathrm{kN}$ in a negative direction of $z$ axis for each foot (see Fig. 2).

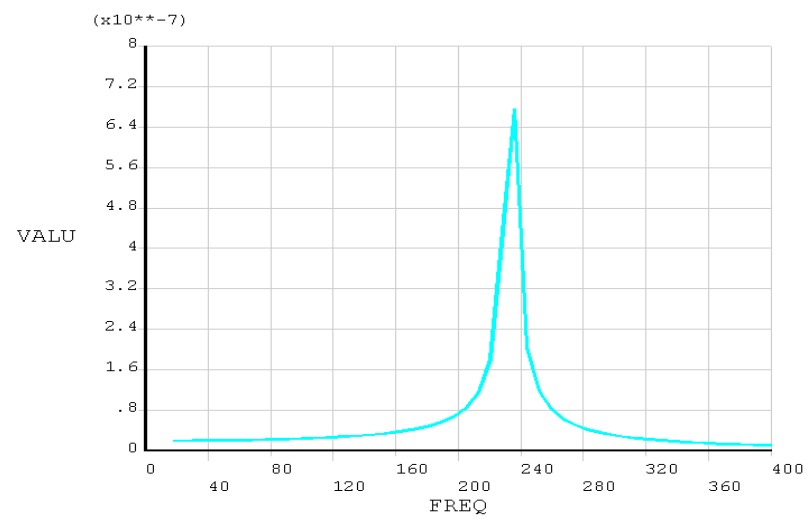

Fig. 5. Vertical deflection in the cyclotron centre (VALU $[\mathrm{m}])$ in dependence on the frequency (FREQ $[\mathrm{Hz}]$ ) of the excitation force (with amplitude of $1000 \mathrm{~N}$ ).

Dependence of a vertical deflection (reminder: harmonic excitation is set in a vertical direction) in the cyclotron centre on the frequency of the excitation force is plotted in Fig. 5. The excitation force amplitude was $1000 \mathrm{~N}$. It is evident that a resonant state occurred at frequency of $236 \mathrm{~Hz}$. Thus during the Cyclone 18 cyclotron operation it is necessary to prevent the possibility of the cyclotron excitation by this frequency.

Table 1 shows the maximum dynamic reaction forces caused by dynamic harmonic loading acting in the places of the cyclotron fixing, which represent mutual cyclotron - floor acting (sum of responses in all four feet is concerned). As already stated, regarding the presupposition (linear behaviour of material, damping, etc.), reaction should be proportional to the chosen amplitude, which is evident in Table 1. Results are specified for excitation frequencies of $50 \mathrm{~Hz}$ (corresponding to the frequencies in the electricity network) and $236 \mathrm{~Hz}$ (resonant).

Before solving the response to the seismic excitation it was necessary to determine natural frequencies and corresponding eigenmodes of the whole system using modal analysis. To solve the response to seismic excitation applying spectral analysis first 10 
eigenmodes were used (first two eigenmodes are shown in Fig. 6). As the excitation is defined up to the frequency of $50 \mathrm{~Hz}$ and the highest considered (tenth) eigenmode belongs to natural frequency of $111.5 \mathrm{~Hz}$, a standard rule for the selection of eigenmodes at this type of analysis, according to which all eigenmodes of the frequencies lower than double the highest excitation frequency should be considered, is fulfilled.

Table 1. Maximum dynamic reaction forces $[\mathrm{N}]$ acting in the cyclotron feet for various frequencies and amplitudes of the excitation force.

\begin{tabular}{|c|c|c|c|}
\hline \multirow{2}{*}{} & \multicolumn{2}{|c|}{ Frequency of the excitation force } \\
\cline { 3 - 4 } & $1000 \mathrm{~N}$ & $\mathbf{5 0 ~ H z}$ & $\mathbf{2 3 6 ~ H z}$ \\
\hline \multirow{3}{*}{$\begin{array}{c}\text { Amplitude of the } \\
\text { excitation force }\end{array}$} & $10000 \mathrm{~N}$ & 1700 & 50900 \\
\cline { 2 - 4 } & $20000 \mathrm{~N}$ & 17000 & 508700 \\
\cline { 2 - 4 } & & 33850 & 1017400 \\
\hline
\end{tabular}
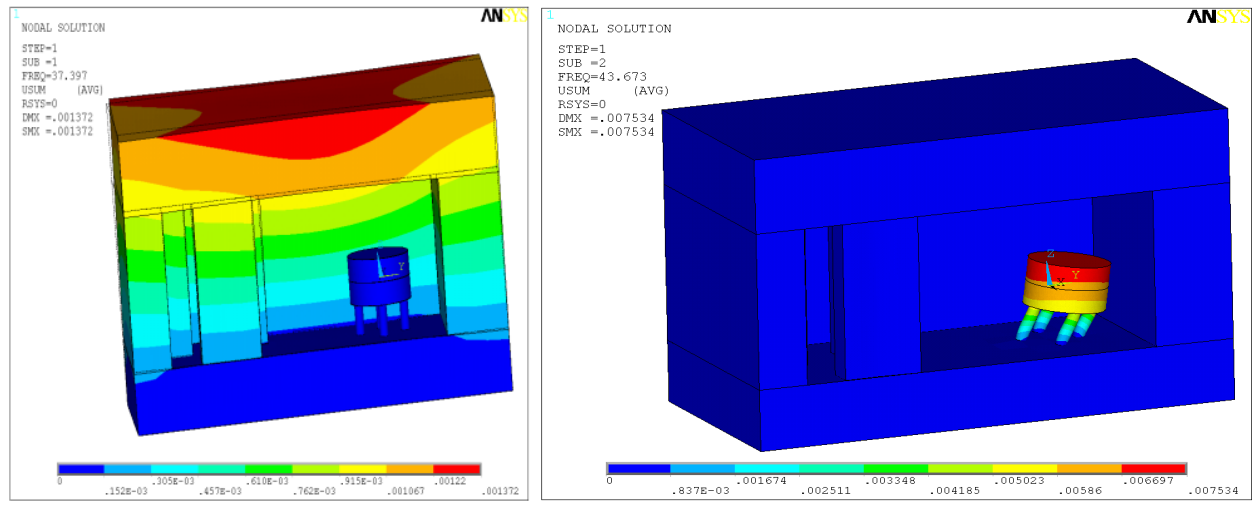

Fig. 6. The first eigenmode (left, $f_{1}=37.4 \mathrm{~Hz}$ ) and the second eigenmode (right, $f_{2}=43.7 \mathrm{~Hz}$ ) of the calculation model of the cyclotron and its chamber.

Table 2. Maximum dynamic reaction force $[\mathrm{N}]$ in individual feet at seismic excitation.

\begin{tabular}{|c|c|c|c|}
\hline Feet (see Fig. 2) & $\boldsymbol{F}_{\boldsymbol{x}}[\mathbf{N}]$ & $\boldsymbol{F}_{\boldsymbol{y}}[\mathbf{N}]$ & $\boldsymbol{F}_{\boldsymbol{z}}[\mathbf{N}]$ \\
\hline $\mathbf{1}$ & 58950 & 30750 & 304250 \\
\hline $\mathbf{2}$ & 61000 & 31100 & 309400 \\
\hline $\mathbf{3}$ & 60000 & 30400 & 299000 \\
\hline $\mathbf{4}$ & 54400 & 22850 & 267500 \\
\hline
\end{tabular}

Results of spectral analysis at the set seismic excitation can be seen in Table 2 and Fig. 7. Table 2 shows the maximum values of dynamic reaction forces acting in the individual cyclotron feet. Fig. 7 represents 2 various views of the computation model with depicted absolute displacements. 


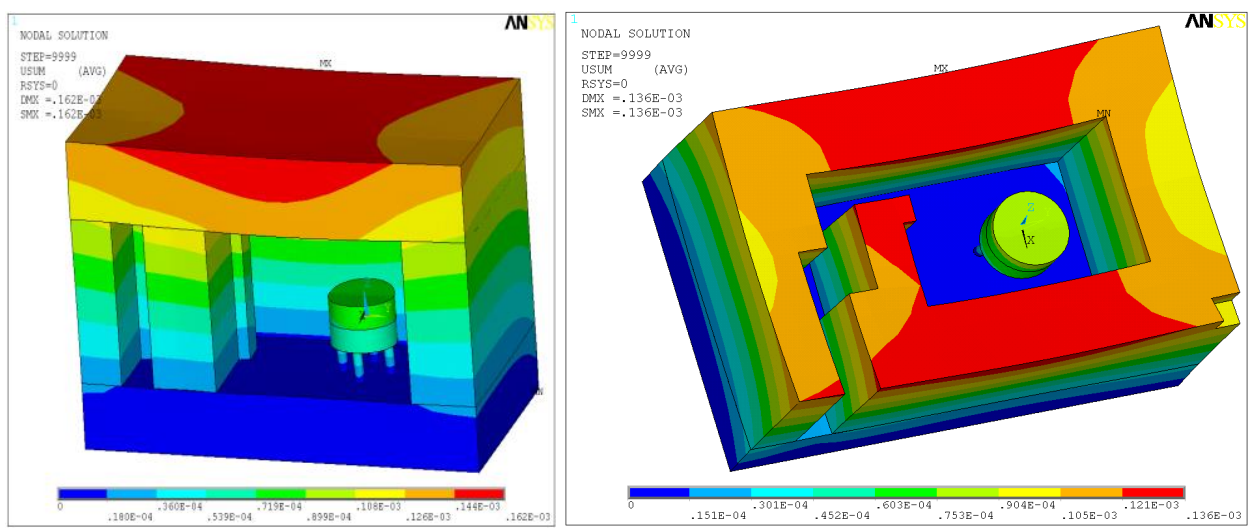

Fig. 7. Dynamic response of the calculation model of the cyclotron and its chamber in the case of seismic excitation.

\section{Conclusions}

The paper presents description of the calculation model of the Cyclone 18 cyclotron and its concrete chamber. The calculation model was created using the ANSYS software on the basis of the finite element method. The created model is parametric, which enables to perform operational calculations with the cyclotron modified parameters (e.g. at optimizing its selected properties). Two loading modes of the cyclotron are simulated: excitation by harmonic sinusoidal force and the seismic excitation.

As a result of the calculation analysis, dangerous excitation frequency of $236 \mathrm{~Hz}$, which causes a resonant vibration of the cyclotron (in vertical direction), was identified. On the basis of the results of the calculations with the model seismic excitation it would be possible to perform the strength and the life analyses of both the cyclotron and the chamber.

The introduced method of the investigation of the cyclotron dynamic acting on the building construction can be generalized to any technological equipment.

The paper has originated in the framework of institutional support for the long-time conception development of the research institution provided by the Ministry of Industry and Trade of the Czech Republic to Research and Testing Institute Pilsen.

\section{References}

1. R.F. Drenick, P.C. Wang, C.B. Yun, A.J. Philappacopoulos, Nucl. Eng. Des. 59, 425 (1980)

2. S. Yoshimura, K. Kobayashi, H. Akiba, S. Suzuki, M. Ogino, J. Nucl. Sci. Technol. 52, 546 (2014)

3. N. Ile, A. Frau, Nucl. Eng. Des. 314, 238 (2017)

4. D.M.S. Ronden, M. A. Henderson, B. Becket, T. Bigelow, J. Caughman, C. Darbos, F. Gandini, C. Nazare, D. Rasmussen, V. Udintsev, Fusion Sci. Technol. 59, 718 (2011)

5. A. Mas Sánchez, R. Chavan, M. Gagliardi, T. Goodman, G. Saibene, P. Santos Silva, M. Vagnoni, Fusion Eng. Des. 146, 1373 (2019)

6. A. Mas Sánchez, G. Aiello, R. Chavan, M. Gagliardi, T. Goodman, M. Henderson, J.D. Landis, G. Saibene, P. Santos Silva, A. Vaccaro, Fusion Eng. Des. 123, 458 (2017) 